\title{
The 3-Dimensional Distribution of Interstellar Dust
}

\author{
Jayant Murthy \\ Indian Institute of Astrophysics \\ email: jmurthy@yahoo.com
}

\begin{abstract}
A knowledge of the three dimensional distribution of interstellar dust is critical in interpreting all observations of the sky, particularly in the understanding of the structure and morphology of our Galaxy. It has been much easier to map the integrated dust extinction through the Galaxy, which is needed in modeling extragalactic sources, but this yields an overestimate of reddening to Galactic objects. Massive surveys, such as Gaia, present both a problem in that the distribution of interstellar dust must be known in order to model the internal structure of the Galaxy and an opportunity in that multi-color data may be used to deconvolve the dust distribution. I will present the current state of the modeling, which is yet in its early stages.
\end{abstract}

Keywords. ISM: clouds, ISM: dust, ISM: extinction, ISM: structure

\section{Introduction}

An appreciation of the importance of the interstellar medium came relatively late in astronomy. The first models of the Galaxy simply counted stars and placed fainter stars at greater distances and, as a result, found that the Galaxy was roughly spherical with the Sun at the center (Fig. 1 - Herschel (1785)). It was not until 1904 that firm evidence for interstellar gas was found by Hartmann (1904) who observed stationary lines in the spectrum of the spectroscopic binary $\delta$ Cep which he postulated as being due to a cloud of gas between us and the star. Even as late as 1927, when Barnard published (posthumously) his catalog of dark nebulae in A photographic atlas of selected regions of the Milky Way (Barnard, Frost, \& Calvert 1927), it was not widely accepted that these were dark clouds of gas and dust rather than non-luminous objects. Certainly, the observational evidence from star counts that the Sun was at the center of the Galaxy Kapteyn (1922) was used by Curtis in the Great Debate between Curtis and Shapley (Trimble 1995) to show that Shapley's globular cluster results (Shapley 1918) were wrong. Conclusive proof for the existence of an all-pervasive interstellar medium was presented by Trumpler (1930a) through a comparison of the angular diameter of open clusters with their photometric magnitude and he found a remarkably modern value of 0.7 magnitudes of extinction for every kiloparsec and it was realized that star counts had to take interstellar absorption into account.

With the advent of large scale surveys such as Gaia (Perryman et al. 2001), an understanding of the three-dimensional distribution and the nature of the interstellar extinction becomes critical to developing models of the Milky Way, particularly in the Galactic disk where not only many of the interesting sources but also most of the dust lies. In this paper, I will discuss the techniques for determining the dust distribution and the concomitant interstellar absorption and the inconclusive results to date. 


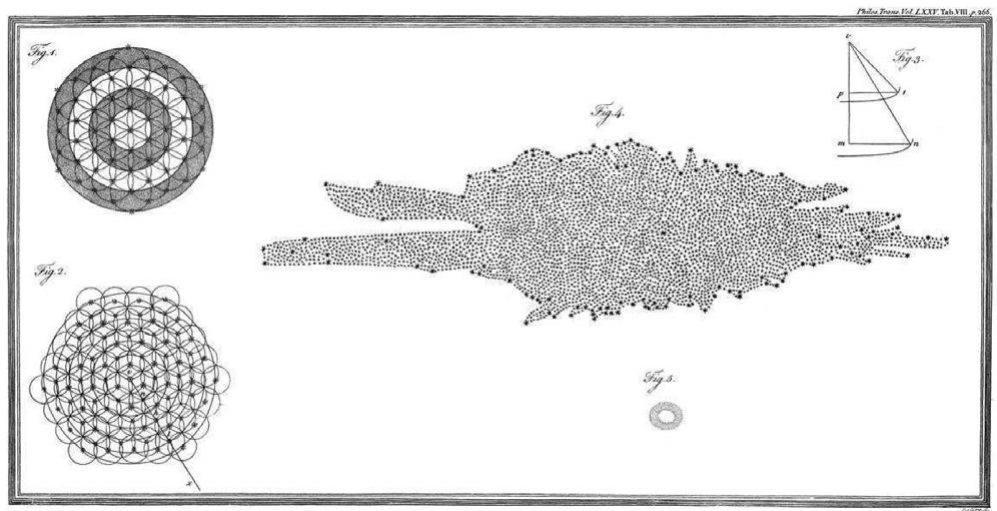

Figure 1. William and Catherine Herschel's model of the Galaxy (Herschel 1785). The Sun is at the center with the distance to stars defined by their brightness. Interstellar extinction was unknown at the time.

\section{Techniques}

The most direct way to observe interstellar dust is through extinction observations as first demonstrated by Trumpler (1930b) with a spectrograph at the Lick Observatory. The spectra of two stars - the target star and a standard star of the same spectral type with no (or little) extinction - are compared with the difference being due to extinction from interstellar dust. Studies of the extinction curve flowered after the launch of the International Ultraviolet Explorer (IUE) to the point where the extinction curve in the ultraviolet (UV) is better defined than in any other wavelength (Fitzpatrick \& Massa 2009), even though the UV data can only be obtained from space. I have shown a range of extinction curves in Fig. 2 normalized to the visible part of the curve. Although there is not much variation in the NIR and the visible, there is little consistency in the UV part of the curve, where most of the structure is. Fitzpatrick \& Massa (1988) have modeled the extinction curve with 5 parameters which Cardelli, Clayton, \& Mathis (1989) found to be dependent solely on $R_{V}\left(=A_{V} / E(B-V)\right)$ raising the hope that extinction at any wavelength could be corrected for through readily derived observational parameters (Fig. 3). More recent results (Fitzpatrick \& Massa 2007) show that the situation is more complex and that there is no typical extinction curve; i.e., the extinction has to be determined separately for every line of sight.

I have summarized the different techniques in deriving the three-dimensional dust distribution in Table 1. In principle, extinction surveys are the only method to directly derive the three-dimensional distribution of interstellar dust as described in the pioneering work by Neckel \& Klare (1980). They divided the sky into 30 areas and correlated the extinction of individual stars with the distance to those stars (Fig. 4) thereby clearly identifying the broad distribution of the dust. This method is restricted to those directions with stellar spectra and distances and hence yields a sparse map of the dust, both across the sky and in depth. More modern attempts use Strömgren 4 color photometry of a given field (e.g. Knude 1979; Franco 2012) to yield precise classification of the stars and the interstellar reddening.

On the other hand, the amount of dust is highly correlated with the column density of neutral hydrogen (H I) (Bohlin, Savage, \& Drake 1978) and $21 \mathrm{~cm}$ surveys exist at high spatial resolution and with depth information from the rotational velocity of the gas. This was exploited by Burstein \& Heiles (1982) who used a combination of reddening and $21 \mathrm{~cm}$ surveys to map the integrated extinction through the Galaxy at a resolution 


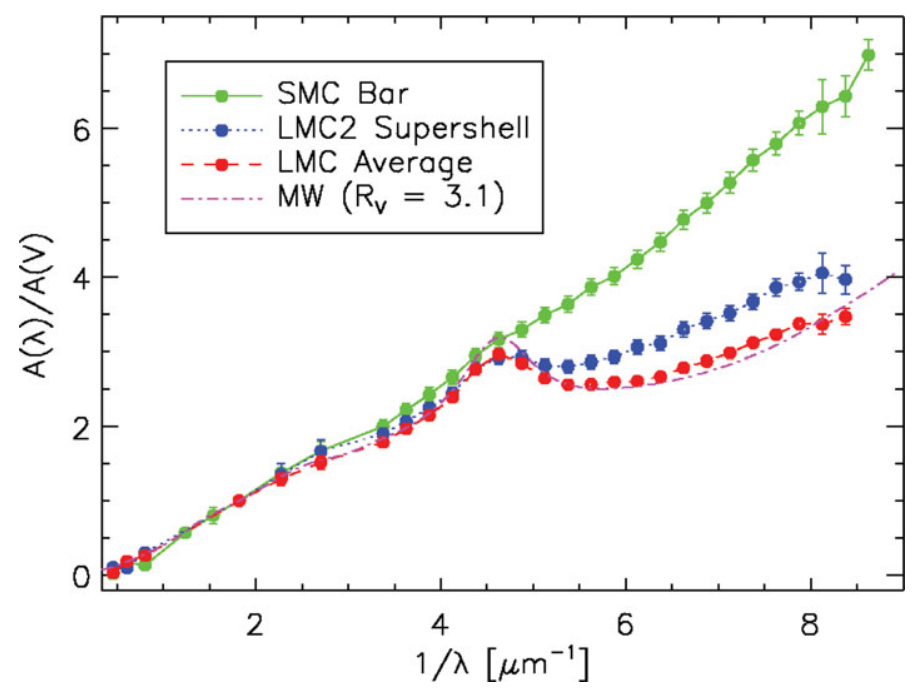

Figure 2. Extinction curves from a variety of different environments (Karl Gordon: personal communication).

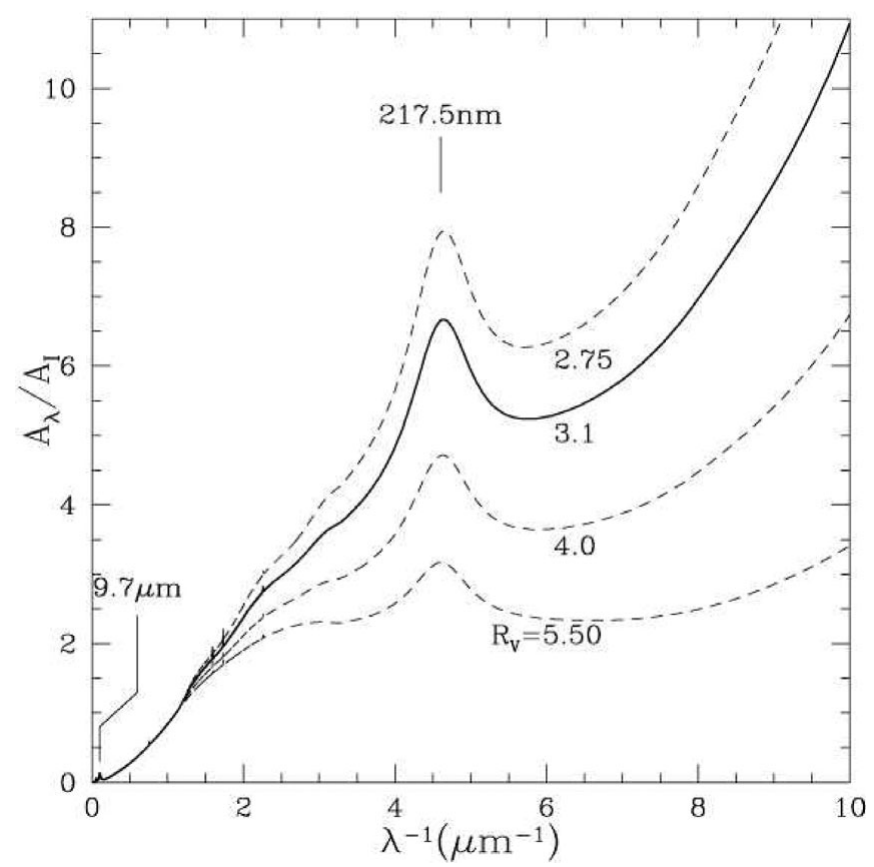

Figure 3. The extinction curve plotted as a function of $R_{V}\left(=A_{V} / E(B-V)\right)$ from Draine (2001). Larger values of $\mathrm{R}$ indicate a large population of grains, as found in dense clouds.

of a few arcminutes and a sensitivity to extinction of about 0.02 magnitudes in $\mathrm{E}(\mathrm{B}-\mathrm{V})$. These were heavily used for almost 20 years with almost 2,000 citations until the easy access of IR data from the Infrared Astronomy Satellite (IRAS).

IRAS observed diffuse emission from interstellar dust over the entire sky (Low et al. 1984) in 4 bands $(12,25,60$, and $100 \mu \mathrm{m})$, where the emission in the longer wavelength bands was due to thermal emission from large grains at a temperature of about $20 \mathrm{~K}$ and that at 12 and $25 \mu \mathrm{m}$ was due to stochastic emission from small grains or large 
Table 1. Methods of deriving extinction

\begin{tabular}{|c|c|}
\hline Method & Notes \\
\hline $\begin{array}{l}\text { Extinction from comparing target star } \\
\text { with either standard stars or models. }\end{array}$ & $\begin{array}{l}\text { Spectral type and distance may be deduced from } \\
\text { spectra. } \\
\text { Depends on availability of suitable stars and so } \\
\text { may be irregularly sampled. } \\
\text { Require spectrum. }\end{array}$ \\
\hline $\begin{array}{l}\text { Band photometry including Strömgren } \\
\text { photometry. }\end{array}$ & $\begin{array}{l}\text { Modern surveys provide photometry of large num- } \\
\text { ber of sources. } \\
\text { May be confused by non-standard extinction laws. } \\
\text { May not produce unique matches. }\end{array}$ \\
\hline Absorption line spectroscopy. & $\begin{array}{l}\text { Measures gas column density rather than dust. } \\
\text { Gas-to-dust ratio may vary. } \\
\text { Sensitive to low density regions. }\end{array}$ \\
\hline IR emission from dust. & $\begin{array}{l}\text { Measures total column density. } \\
\text { May be affected by different phases of the ISM in } \\
\text { the line of sight. }\end{array}$ \\
\hline $21 \mathrm{~cm}$ surveys. & $\begin{array}{l}\text { Measure of gas density. } \\
\text { Some distance information from rotational veloc- } \\
\text { ity but poor distance resolution. }\end{array}$ \\
\hline Star counts. & $\begin{array}{l}\text { Useful to map dust in dense clouds (IR observa- } \\
\text { tions). } \\
\text { Require sufficient star density to overcome statis- } \\
\text { tical issues. }\end{array}$ \\
\hline
\end{tabular}

molecules; and the $60 \mu \mathrm{m}$ emission is from a mix of both small and large grain emission. The extinction throughout the Galaxy was calculated by Schlegel, Finkbeiner, \& Davis (1998) from the $100 \mu \mathrm{m}$ emission modified by the temperature of the grains. This became an immediate success as they provided a map of the sky with tools to derive the extinction in any line of sight and there is now a cottage industry in finding locations where their map is less accurate than in others. The SFD maps appear to overestimate the extinction by about $15 \%$ in regions of high extinction.

These maps are still 2-dimensional and only represent the total extinction along the line of sight. As such, they are valuable in interpreting extragalactic observations but are less useful in estimating the amount of extinction within our Galaxy. $21 \mathrm{~cm}$ maps, which are highly correlated with the $100 \mu \mathrm{m}$ emission, include broad distance information through the rotational velocity of the gas. However, these can only trace large-scale features. The only way of obtaining detailed extinction measurements on the small scale remains observations of individual stars, preferably the extinction directly but, if not possible, then the observations of absorption lines in the ISM. In the next section, we will detail the several attempts to define this, particularly with the large amount of data now becoming available.

Many of the attempts to derive the 3-dimensional distribution use star counts. The essence of this is to compare an observed stellar distribution as a function of distance with a model of the stellar distribution, such as the Besançon model (Robin et al. 2003). These are statistical in nature and may not be a good representation of the interstellar structure in any given volume of our Galaxy. Star counts are also valuable in studies of selected regions of the sky. By choosing the spectral range carefully, different column densities of dust may be probed. For instance, one of the only ways of defining the structure in dense molecular clouds is through IR mapping, where the star density is high and the cross-section of the dust grains is low. For instance, Lada, Alves, \& Lada (1999) were able to achieve a spatial resolution close to an arc minute in their study of the IC 5146 


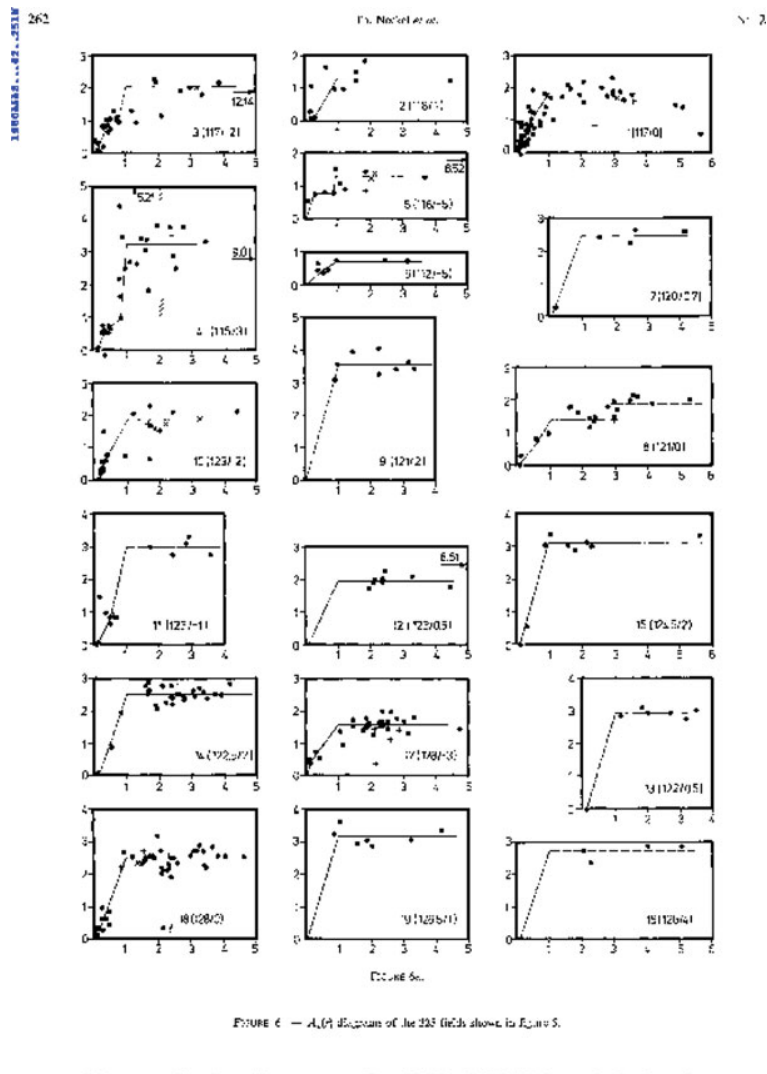

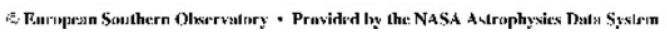

Figure 4. A sample of extinction maps from Neckel \& Klare 1980. Note the distribution of the dust into large clouds and empty voids.

dark cloud. Observations in the visible and, more so, in the ultraviolet are sensitive to much lower amounts of dust but are limited because of the lower star density.

\section{Extinction maps}

One of the difficulties in understanding the three-dimensional structure of the interstellar medium is that structure is found on all scales, including between two stars of a binary system (Meyer \& Blades 1996). The very shortest lines of sight, within the Local Interstellar Medium (LISM) are difficult to detect through extinction studies but have been probed through absorption line spectroscopy (e.g.. Redfield \& Linsky 2008; Welsh et al. 2010) and, even within 15 pc of the Sun, Linsky \& Redfield (2009) have found 15 clouds. The column densities are low, less than $10^{19} \mathrm{~cm}^{-2}$ corresponding to an extinction $\left(\mathrm{A}_{V}\right.$ of less than 0.01 magnitudes, and so have little effect on the total extinction at larger scales.

Extinction measurements become possible on larger scales and Vergely et al. (2010) have mapped the dust within $250 \mathrm{pc}$ of the Sun finding an excellent correlation with absorption line measurements of neutral sodium (Na). Notably, they found that the Local Cavity, the large volume of hot gas around the Sun, is present both in the gas and the dust. However, they also found that the opacity was higher in the dust in the Northern 
Table 2. Extinction models and maps

\begin{tabular}{|c|c|c|}
\hline Reference & Method & Notes \\
\hline $\begin{array}{l}\text { Neckel \& Klare } \\
\quad(1980)\end{array}$ & $\begin{array}{l}\text { Strömgren photometry of } 11,000 \\
\text { stars }\end{array}$ & Maps provided in Galactic plane \\
\hline $\begin{array}{l}\text { Burstein \& Heiles } \\
\quad(1982)\end{array}$ & $21 \mathrm{~cm}+$ galaxy counts & Integrated extinction \\
\hline $\begin{array}{l}\text { Arenou, Grenon, \& } \\
\text { Gómez (1992) }\end{array}$ & $\begin{array}{l}\text { Tridimensional model based on } \\
\text { photometric data. }\end{array}$ & $\begin{array}{l}\text { Python code at } \\
\text { https://github.com/alsmirn/ } \\
\text { adist/blob/master/extinction/arenou.py }\end{array}$ \\
\hline Hakkila et al. (1997) & $\begin{array}{l}\text { Modeling based on extinction } \\
\text { observations. }\end{array}$ & $\begin{array}{l}\text { Fortran code at http://asterisk.apod. } \\
\quad \text { com/view topic.php?f }=35 \& \mathrm{t}=29300\end{array}$ \\
\hline $\begin{array}{l}\text { Méndez \& van Altena } \\
\quad(1998)\end{array}$ & Modeling of reddening surveys. & Code available from authors. \\
\hline Schlegel et al. (1998) & $\begin{array}{l}\text { Total extinction from IRAS } \\
\text { observations. }\end{array}$ & Many overestimate extinction by up to $14 \%$ \\
\hline Drimmel et al. (2003) & $\begin{array}{l}\text { Based on SFD maps + dust } \\
\text { model }\end{array}$ & $\begin{array}{l}\text { IDL code at ftp://ftp.to.astro.it/ } \\
\text { astrometria/extinction/ }\end{array}$ \\
\hline Dobashi et al. (2005) & Star counts from DSS & Catalog of dark clouds \\
\hline $\begin{array}{l}\text { Amôres \& Lépine } \\
(2005)\end{array}$ & $\begin{array}{l}\text { Models based on } 21 \mathrm{~cm}+\text { IRAS } \\
\text { data }\end{array}$ & $\begin{array}{l}\text { Description and code at http://www.astro. } \\
\text { iag.usp.br/ amores/modextin.html }\end{array}$ \\
\hline Marshall et al. (2006) & 2MASS + Besançon model & $\begin{array}{l}\text { Available from http://cdsweb.u-strasbg. } \\
\text { fr/cgi-bin/ qcat?J/A+A/453/635 }\end{array}$ \\
\hline Sale et al. 2009 & Modeling of IPHAS data & Focuses on model. \\
\hline Vergely et al. (2010) & $\begin{array}{l}\text { Extinction in the Solar } \\
\text { neighborhood. }\end{array}$ & Data cube at 5 pc resolution \\
\hline Gontcharov (2010) & 2MASS + Hipparcos & \\
\hline $\begin{array}{l}\text { Jones, West, \& Foster } \\
\text { (2011) }\end{array}$ & SDSS spectra of M dwarfs & $\begin{array}{l}\text { Available from http://people.bu.edu/ } \\
\text { aawest/dust.html }\end{array}$ \\
\hline $\begin{array}{l}\text { Majewski, Zasowski, } \\
\text { \& Nidever }(2011)\end{array}$ & 2MASS + Spitzer photometry & \\
\hline Dobashi (2011) & 2MASS color excess & Catalog of dark clouds \\
\hline Gontcharov (2012) & 2MASS + Hipparcos photometry & \\
\hline Gontcharov (2013) & 2MASS + WISE photometry & Spatial variations of Extinction Law \\
\hline $\begin{array}{l}\text { Kohyama et al. } \\
\text { (2013) }\end{array}$ & $\begin{array}{l}\text { total extinction from IRAS } \\
\text { emission }\end{array}$ & \\
\hline Sale (2012) & Bayesian models & Modeling only \\
\hline
\end{tabular}

hemisphere as compared to the Southern, an asymmetry not seen in the gas. This implies that the gas to dust ratio is higher in the Northern hemisphere, a conclusion also drawn by (Knude \& Høg 1999). This is troubling for large scale extinction maps because most have the assumption, either implicitly or explicitly, that the dust properties are uniform throughout the Galaxy.

Most efforts to measure the extinction in three dimensions on large scales have used photometric surveys. I have already mentioned the work by Neckel \& Klare (1980) who used archival UBV $+\mathrm{H}_{\beta}$ data for more than 11,000 stars to trace the global dust distribution. Although, or perhaps because, their data were sparse, they found that the dust was distributed in steps indicating dense clouds with large volumes of empty space. More recent work (summarized in Table 1) has used much larger surveys with correspondingly better resolution. These have been purely empirical determinations of the interstellar distribution. A different approach is to use models of the Galaxy and to fit the extinction to the Galactic structure assuming that the gas and dust are well-mixed and therefore that the extinction is closely tied to the gas. The advantage of these models (summarized in Table 2) is that they tie the extinction map to a physical model of the Galaxy but, perhaps, at the cost of fitting the small scale structure of the dust.

\section{Summary}

Trying to predict the 3-dimensional extinction over any part of the sky is a complicated problem that may not be easily resolved. The distribution of the dust (and gas) is clumpy 
on the smallest scales with a structure not easily resolvable by observations. Because of the difficulty in observing the dust directly and in determining the distance to the dust, we are forced to rely upon background stars which are distributed unevenly and, depending on the wavelength and the position in the sky, may be relatively small in number. Most studies also assume that the dust properties are the same over the entire galaxy, and then proceed to derive differences from the standard laws. In all likelihood, these represent actual differences in the dust properties, such as the dust-to-ratio. Actually determining the extinction to a particular object, rather than a general statistical distribution, is likely best done by a detailed examination of observations around that object, particularly using IUE observations, if available.

It is also difficult to represent the distribution in a manner that can be readily applied to the problem at hand. Fitzpatrick \& Massa (2007) suggested that the most accurate method was to derive the extinction from a nearby star observed by the IUE satellite. This is not practical for most large scale surveys for which the only solution is to use a model-based approach such as Drimmel \& Schlegel (2001) or Amôres \& Lepine (2005). The current crop of large scale surveys both provides a challenge to 3-D models of the dust and an opportunity to improve those models. In particular, I wish to highlight two results presented at this meeting: Schlafly et al. (2013) who has used the PanStarrs survey to derive unprecedented views of the structure of the ISM in selected areas and the Chinese Stromgren photometry survey Wang et al. (2013), to a depth of 21 mag.

\section{References}

Amôres \& Lépine 2005 AJ 130, 679

Arenou, F., Grenon, M., \& Gómez 1992 A\&SA 258, 104

Barnard, E. E., Frost, E. B., \& Calvert, M. R. 1927, [Washington] Carnegie institution of Washington, 1927

Bohlin, Savage, \& Drake 1978 ApJ 224, 132

Burstein, D. \& Heiles, C. $1982 A J$ 87, 1165

Cardelli, J. A., Clayton, G. C., \& Mathis, J. S. 1989 ApJ 345, 245

Dobashi, K., Uehara, H., Kandori, R., Sakurai, T., Kaiden, M., Umemoto, T., \& Sato, F. 2005 PASJ 57, S1

Dobashi, K. 2011 PASJ 63, S1

Draine, B. T. 2001 Encyclopedia of Astronomy and Astrophysics, Ed. P. Murdin, Taylor E Francis 1266

Drimmel, R., Cabrera-Lavers, A., \& López-Corredoira, M. 2003 A $\& A$ 409, 205

Fitzpatrick, E. L. \& Massa, D. 1988 ApJ 328, 734

Fitzpatrick, E. L. \& Massa, D. 2007 ApJ 663, 320

Fitzpatrick, E. L. \& Massa, D. 2009 ApJ 699, 1209

Franco, G. 2012 A\&A 543, 39

Gontcharov, G. A. 2010 Astr. Lett 36, 584

Gontcharov, G. A. 2012 Astr. Lett. 38, 87

Gontcharov, G. A. 2013 Astr. Lett. 39, 83

Hakkila, J, Myers, J. M., Stidham, B. J., \& Hartmann, D. H. 1997 AJ 114, 2043

Hartmann, J. 1904, ApJ, 19, 268

Herschel, W. 1785, Phil. Trans. R. Soc. Lond., 75, 213

Jones, D. O., West, A. A., \& Foster, J. B. 2011 AJ 142, 44

Kapteyn, J. C. 1922 ApJ, 55, 302

Knude, J. 1979 A $\& A 71,344$

Knude, J. \& Høg, E. 1999 A\&A 341, 451

Kohyama, T., Shibai, H., Fukagawa, M., \& Sumi, T. 2013 PASJ 65, 13

Lada, C. J., Alves, J., \& Lada, E. A. 1999 ApJ 512, 250 
Linsky, J. L. \& Redfield, S. 2009 AIP Conf. Proc. 1156, 174

Low, F. J., Young, E, Beintema, D. A., Gautier, T. N., Beichman, C. A., Aumann, H., H., Gillett, F. C., Neugebauer, G., Boggess, N., \& Emerson, J. P. 1984 ApJL 278, 19L

Majewski, S. R., Zasowski, G., \& Nidever, D. L. 2011 ApJ 739, 25

Marshall, D. J., Robin, A. C., Reylé, C., Schultheis, M. \& Picaud, S. 2006 A\&A 453, 635

Méndez, R. A. \& van Altena, W. F. 1998 A\& $A$ 330, 910

Meyer, D. M. \& Blades, J. C. 1996 ApJ 464, 179

Neckel, Th. \& Klare, G. A. 1980 A\&A.SS 42, 251

Perryman, M. A. C., de Boer, K. S., Gilmore, G., Høg, E., Lattanzi, M. G., Lindegren, L., Luri, X., Mignard, F., Pace, O., \& de Zeeuw, P. T. 2001 A $\& A 369,339$

Redfield, S. \& Linsky, J. L. 2008 ApJ 673, 283

Robin, A. C., Reylé, C., Derrieè, S., \& Picaud, S. 2003 A $\& A$ 409, 523

Sale, S. E., Drew, J. E., Unruh, Y. C., Irwin, M. J., Knigge, C., Phillipps, S., Zijlstra, A. A., Gänsicke, B. T., Greimel, R., Groot, P. J., Mampaso, A., Morris, R. A. H., Napiwotzki, R., Steeghs, D., \& Walton, N. A. 2009 MNRAS 402, 713

Sale, S. E. 2012 MNRAS 427, 2119

Schlafly, E., Green, G., Finkbeiner, D., \& Rix, H.-W. 2013 this conference

Schlegel, D. J., Finkbeiner, D. P., \& Davis, M. 1998 ApJ 500, 525

Shapley, H. 1918 PASP 30, 42

Trimble, V. 1995 PASP, 107, 1133

Trumpler, R. 1930 PASP, 42, 214

Trumpler, R. 1930 PASP, 42, 267

Vergely, J.-L., Valette, B., Lallement, R., \& Raimond, S. 2010 A\&A 518, 31

Wang, W., Zhao, G., Chen, Y., Liu, Y., Ma, L., \& Mendez, R. 2013 this conference

Welsh, B. Y., Lallement, R., Vergely, J.-L., \& Raimond, S. 2010 A $\& A$ 510, 54

\section{Discussion}

AndRÉ Martins: Which model is to be preferred for extinction of specific regions? In particular, how about Drimmel's model?

JAYANT MURThY: Extinction in specific regions is always difficult and none of the models are likely to yield a satisfactory answer. They may also vary in their applicability to specific regions. I have tabulated many of the models in the paper. 